\title{
Cloud Computing: The Future of IT INDUSTRY
}

\author{
Munther Abualkibash and Khaled Elleithy \\ Department of Computer Science, University of Bridgeport, Bridgeport, CT, USA \\ mabualki@bridgeport.edu, elleithy@bridgeport.edu
}

\begin{abstract}
As a result of the research processing in the computing field, a new computing model appeared based on the development of many computing models such as parallel computing, distributed computing, and grid computing. Many normal distributed computers collaborate of achieve a function like a super computer. The computation will be assigned to this super computer rather than local computer or remote server. This is the basic concept of cloud computing. However, there is a new implementation of cloud computing was introduced based on using the internet millions of computers connected to a super cloud. Cloud computing has several advantages such as; user does not need to worry about how the cloud runs, viruses, maintenance, etc. We would expect that cloud computing is going to reshape the IT industry. In this paper we discuss cloud computing from different angles such as concept, characteristics and classifications of cloud computing.
\end{abstract}

\section{KEYWORDS}

Cloud computing and grid computing.

\section{INTRODUCTION}

Now a days, cloud computing has become widely liked. Cloud services provided by many companies such as Amazon, Google, IBM, Yahoo and Microsoft, especially for business customers. These services installed on cloud provider's virtualized servers are approached over the Internet. Many companies like using these services, without any need to own and maintain server infrastructure. The major differences of cloud computing to the classic rented servers are the contracts and payment models. In cloud services, user pays relying on used resources, i.e. CPU-hours, data storage. Purchasing resources is determined by current need.

Cloud computing makes virtual server infrastructures available for companies. The intentional advantage is that business organizations do not have to buy their own hardware to make services available for their customers. Therefore, end users use derived service instead of raw cloud service [1].

\section{WHAT IS CLOUD COMPUTING?}

Cloud computing is not consisting of one part concept, it is a theoretical term. Various cloud providers make different services available. To understand cloud computing, we have to show the difference between four kinds of cloud services that exist at this time:

DOI : $10.5121 /$ ijdps.2012.3401 
1. Infrastructure as a Service (IaaS), provides low-level services like virtual machines which can be booted with a user-defined hard disk image, i.e. Amazon EC2 [2]. Virtual hard disks that can be accessed from different virtual machines are another example of infrastructure as a service.

2. Platform as a Service (PaaS) means that the cloud operator provides an API which can be used by an application developer to develop "number-crunching" applications or web applications with friendly user-interfaces. An example of Paas is Google's App Engine [3].

3. Software as a Service (SaaS) is useful for end-users. Examples are web-based office applications like Google Docs or Calendar [1].

4. Hardware as a Service (HaaS), this model is beneficial to the business organization users, since they do not need to commit in creating and taking care of data centers [8].

Clouds are a large group of virtualized resources able and very convenient to be used such as hardware, development platforms and/or services. These resources characterized by continuously and slightly restructure in order to achieve the desired variable load (scale), giving also the best act of using resources [4].

\section{What IS A GRID COMPUTING?}

The definition of the grid as a set of resources coordinated to work together as parts of a mechanism or an interconnecting network not subjected to centralized control to provide a service with significant quality that is not quick and easy to accomplish normally in other systems, by using an open and standard interfaces and general purpose protocols. [5].

\subsection{Differences between cloud and grid}

\subsubsection{Resource Sharing}

Grids provide an improved way of resource pooling to guarantee sharing resources fairly, from one side of business organizations to the other [4], [14]. However, clouds make the resources that the service provider requires available on demand [4].

\subsubsection{Heterogeneity}

One of the challenges is to find ways to establish an area data intensive programming and scheduling framework in heterogeneous [13]. Heterogeneous hardware as well as software resources are assembled and maintained the gather by both cloud and grid models [4].

\subsubsection{Virtualization}

Resource heterogeneity supported by grid services with interfaces, a large individual resource pool consists of virtualized sum of parts. Therefore, Virtualization covers data and computing resources [6]. But cloud Computing adds the virtualization of hardware resources [4].

\subsubsection{Security}

Virtualization and security are associated, since virtualization makes the complete environments separation possible. Therefore, in clouds each user has his access to an individual virtualized environment different than other user. However, end user security is not that big issue in grids [4], [7]. Furthermore, in a Virtual Organization all the available resources can be accessed by authorized representative as part of security services [4] [6]. 


\subsubsection{High Level Services}

Data transfer and metadata search are some of a few services provided by grid. In case of cloud, it is badly effected of an absence of some high level services, which is somehow caused because of the lower level of maturity of the paradigm. As a result, this kind of problems can be handled at the application level [4].

\subsubsection{Architecture, Dependencies and Platform Awareness}

Virtualization gives power to cloud applications architecture. But grids accept only grid applications, thus imposing hard requirements to the developers [4].

\subsubsection{Software Workflow}

Service and job oriented are intrinsic nature of grids; they strongly suggest the truth of the need to execute in the proper the integration of the services workflow and location which is not necessary in case of on demand deployment as the one in the clouds. [4].

\subsubsection{Scalability and Self-Management}

Programmers are not controlled of being able to handle any scalability issues in grids and cloud. But in grid, scalability is mostly getting the power by growing larger the amount of working nodes. Nevertheless, clouds give the ability of changing the size of virtualized hardware resources automatically. Plus, a dynamic reconfiguration is necessary in scalability, as the system scales any new requirements to be restructured by the system itself. So selfmanagement as well as scalability is easily done in case of domain with a single administrative, but many problems could be happened outside organization domain. Yet, the whole systems of grids do not have a single owner, that reason will put grids in difficult condition. In the other side, clouds are operated by single companies [4], [15].

\subsubsection{Usability}

Clouds are convenient to use with an easy manner, the deployment details act in state of being concealed from the user [4].

\subsubsection{Standardization}

Grids have done a lot of hard work to succeed in having standardization in the user and the inner interface which dealing with accessing resources. But the user who has permission to approach interface to the cloud which has very often same standard technologies foundation such as in grids, however inner interfaces standardization is having a main serious issue. These internal interfaces are being in concealed condition by the enterprises, thus impede the progress of the ability of communication among different clouds and the fact of being possible of a worldwide organizations union of clouds. Some of the issues of testing the cloud's abilities, like monitoring, storage, Quality of Service, union between different organizations, etc. have been managed at earlier time by grids. Moreover, clouds introduce particular components that call for standardization [4], [16].

\subsubsection{Payment Model}

First attempting of grid was mainly supported by public funding while in the opposite side, cloud has been motivated by commercial offers. Moreover, grid services are charged using a 
stabled rate per service or idle resources shared by various organizations. However, cloud users are usually charged using a pay per-use model [4].

\subsubsection{Quality of Service}

Grids are not giving the best Quality of Service level, due to its way of working by collaborating and based on resource sharing principles. Any grid application, based on top of the grid has to support any service, shall be fulfilled by itself. Mechanisms between infrastructure providers for Service-Level Agreements in the grid have been set. However, Quality of Service existing as an essential feature of many clouds, e.g. By this time, Amazon has tried her best to have a good Quality of service, e.g., 99.9\% infrastructure uptime, by means of basic Service-Level Agreements. Note that Amazon is free of any responsibility in case of power outages, system failures or other interruptions [4].

\subsection{Similarities between grids and clouds}

Grids need to increase the combination of virtualization technologies to get some of the advantages that clouds present by nature, such as, the quality of hardware level of being easily expanded or upgraded. In addition, grids need to make entry points available in easy way to make a wider adoption possible by end users, i.e., grids are a user friendly, virtualized and utility expanded automatically, which shows without doubt a similar characteristics with current clouds. Moreover, many of the existing approaches that merge grids and clouds together, which can also be seen as a union of higher level networking with virtualization developed to a high degree of complexity. Plus, clouds offer a set of features exposed restricted within certain limits [4].

\section{Classification of Cloud Computing}

Many classifications of the cloud computing designed plan can be found, but most were produced and represented according to the companies which offer cloud services for sale and not represented according to enterprise IT, who buys services of cloud and software [8].

\subsection{Cloud Architecture}

Cloud Architecture is the scheme of applications programs used to direct the operation of accessing on- demand service using Internet. They are basic on infrastructure which is used only when it is required that draw the needful resources on-demand and accomplish a specific job, then leave the unnecessary resources and often destroy them after the job has been completed. Figure 1 illustrates cloud architecture.

\begin{tabular}{|c|}
\hline Software-as-a-Service (SaaS) \\
\hline Platform-as-a-Service (PaaS) \\
Developers implementing cloud application \\
\hline Infrastructure-as-a-Service (IaaS) \\
[(Virtualization, Storage Network) as-a-Service] \\
\hline Hardware-as-a-Service \\
\hline
\end{tabular}

Figure 1. Cloud Layered Architecture 
The services are able to be reached all over the world, with the cloud look as a single point of access by consumers for all his computing needs. Cloud Architectures deal with the key difficulties of large scale data processing. Some examples of cloud architecture are processing Pipelines, Batch Processing Systems etc [8].

Basically clouds mode could be defined by four types [8], [12]:

\subsubsection{Private cloud}

Data and processes are controlled within the organization without that much of worrying regarding network bandwidth, security issues and common legal requirements of using cloud services through open and public networks might be required.

\subsubsection{Public cloud}

It explains the traditional way of cloud computing attitudes, through which resources are available on demand, over the Internet, using web applications or web services, from another provider act as third party who help on shares resources.

\subsubsection{Hybrid cloud}

The environment is made up of several internal and/or external providers.

\subsubsection{Community cloud}

Same cloud infrastructure shared by many organizations.

\subsection{Virtualization Management}

It is the technique that removes linking together the hardware and operating system. It directs to the source of the logical resources abstraction away from their physical resources to be more flexible, reduce costs and make a good improvement in business value.

Essentially virtualizations in cloud have so many different types, such as, network virtualization, server virtualization and storage virtualization. Server virtualization can be described as an associating of single physical resources to several logical partitions or representations.

In a virtualized environment, computing environments can be produced in a forceful dynamic manner, enlarged, become smaller or go in a specified direction or manner as demand varies. Virtualization is therefore highly suitable to a dynamic cloud infrastructure, because it provides important advantages in isolation, manageability and sharing [8].

\subsection{Fault Tolerance}

In case of not achieving the desired end, there will be a duplicate copy of instance of the application which is fully prepared to take over without delaying or interrupting the continuity is called failover. A period when Cloud computing service is not available extends into the more very subtle version of cloud service platforms. The main problem for cloud computing is how to reduce any kind of outage failover to provide the trustworthy services [8]. 


\subsection{Security}

Usually security is the most important issue in terms of data, infrastructure and virtualization etc. Collective of information is not only a competitive asset, but it often consist of information of customers, consumers and employees that, in the wrong hands, could create a civil obligation and perhaps criminal charges. Cloud computing can be made secure but securing cloud computing data is a contractual issue as well as a technical one [8].

\subsection{Load Balancing}

Load balancing is often used to perform failover- the state of a service of remaining in a particular condition even after the failure of one or more of its components. The qualities of components are checked always and when one becomes non-responsive, the load balancer is aware and no longer transmits traffic to it. This is inherited feature from grid-based computing for cloud-based platforms. Energy keeping and resource utilized are not always the main issue when talk about cloud computing; however with proper load balancing in place resource utilized can continue to be to a minimum. This is not only serves to maintain costs low and enterprises, it also reduce stress on the circuits of each individual box, making them possible but not yet actual last longer. Load balancing also empowers other important features such as scalability [8].

\subsection{Scalable Data Storage}

Cloud storage empowers client to store data into the cloud without worrying about how it is stored or backing it up. The main issues related to cloud storage are reliability and security. Clients are not likely to entrust their data to another company without a guarantee that they will be able to access their information whenever they want and no one else will be able to get at it [8].

\section{Cloud Challenges}

\subsection{Security and Privacy}

Putting data, running software at someone else's hard disk, and using someone else's CPU appears to be very hard when you think about [12], [13]. In addition, using multi tenancy model created more security issues to be solved, such as, shared resources on the same physical machine, and the other issue is that in cloud good and bad users could share resources and may share the same network address then any bad behaviour will affect them all which is going to damage the reputation of many good users on cloud [12].

\subsection{Costing Model}

Moving towards cloud reduces the infrastructure cost, but at the same time it raises the cost of data communication. In this case, any transactional applications may not be suitable for cloud computing. However, on-demand computing makes sense only for CPU intensive jobs [12].

\subsection{Charging Model}

Cost calculations based on consumptions of static computing, and the unit of cost analysis is an instantiated virtual machine. However, in SaaS cloud providers, the cost they offer for developing multi-tenancy could be very expensive [12]. 


\subsection{Service level agreement}

It is a negotiation between the cloud providers and consumers, to obtain guarantees from providers on service delivery [12].

\subsection{Power}

Cloud computing provides different type of services to satisfy the needs of consumers, huge power is consumed. A smart energy system for resource management is highly recommended [13].

\section{Current Players}

\subsection{Amazon Elastic Compute Cloud (Amazon EC2)}

Amazon Elastic Compute Cloud (Amazon EC2) is a web service that makes the availability of compute capacity to be resizable in the cloud. It is purpose to make web-scale computing much easy for developers. Amazon EC2's simple web service interface gives customers permissions to get and configure capacity with least conflict. It gives customers the ability of the complete control of their computing resources and allows them run on Amazon's computing environment. Amazon EC2 minimize the time needed to get and boot new server instances to minutes, giving customers the ability to scale capacity without delay, both up and down, as their computing requirements change. Amazon EC2 alters the economics of computing by let customers to pay only for capacity that they really use. Amazon EC2 supplies developers the tools to build failure recovery applications and separate themselves from usual failure scenarios [2]. Figure 2 has given an EC2 system use pattern [11].

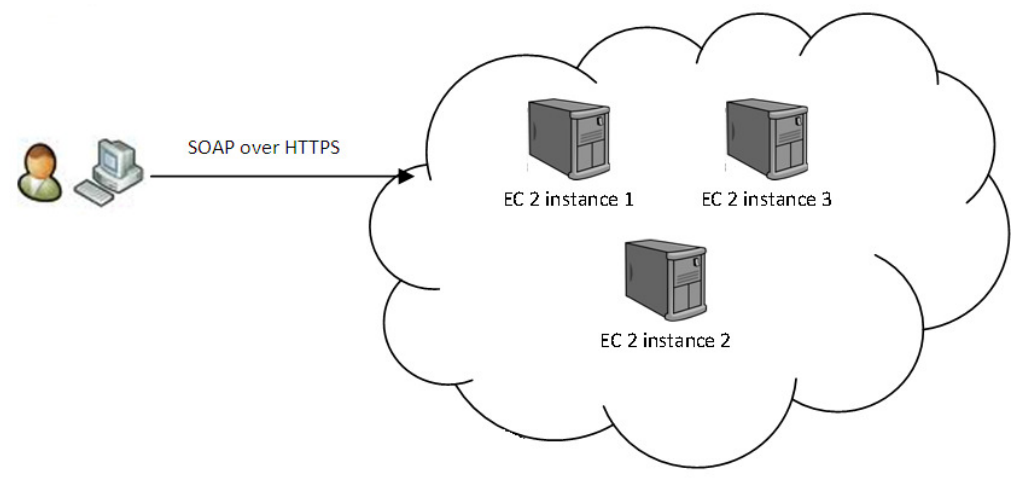

Figure 2. Usage of Amazon Elastic Compute Cloud

\subsubsection{Amazon EC2 Functionality}

Amazon EC2 introduces a reliable virtual computing environment, giving customers permissions to use web service interfaces to start instances with a different type of operating systems, load them with their custom application environment, control the use of their network's access permissions, and operate their image using as many or few systems as they request [2]. 
International Journal of Distributed and Parallel Systems (IJDPS) Vol.3, No.4, July 2012

\subsubsection{Services in Amazon EC2 [2]}

1. Elastic: increase or decrease capacity within minutes, not hours or days.

2. Completely Controlled:

- Complete control of instances.

- Having root access to each one.

3. Flexible: having the choice of multiple instance types, operating systems, and software packages.

4. Designed for use with other Amazon Web Services.

5. Reliable: The Amazon EC2 Service Level Agreement commitment is $99.95 \%$ availability for each Amazon EC2 Region.

6. Secure: provides numerous mechanisms for securing customer computer resources.

7. Inexpensive: paying a very low rate for the compute capacity consumed.

\subsubsection{Features of Amazon EC2 [2]}

1. Amazon Elastic Block Store: offers persistent storage for Amazon EC2 instances.

2. Multiple Locations: place instances in multiple locations.

3. Elastic IP Addresses: static IP addresses designed for dynamic cloud computing associated with the account not a particular instance.

4. Amazon Virtual Private Cloud: secure and seamless bridge between a company's existing IT infrastructure and the Amazon Web Service cloud.

5. Amazon CloudWatch: a web service that provides monitoring for AWS cloud resources.

6. Auto Scaling: scale capacity up or down.

7. Elastic Load Balancing: distributes incoming application traffic across multiple instances.

8. High Performance Computing (HPC) Clusters: Cluster Compute and Cluster GPU Instances have been designed to support high performance network capability.

9. VM Import: virtual machine images will be imported from an existing environment to Amazon EC2 instances.

\subsection{Microsoft Windows Azure}

\subsubsection{OVERVIEW}

Microsoft Windows Azure is based on cloud computing. Running applications and storing data on machines in data center accessed via the internet can provide a lot of advantages. Yet wherever customers run, applications are incorporate as part of platform. For on-premises applications, such as customers running in the inner side of an organization's data center, this platform normally contains an operating system used to store data and possibly more. Applications running in the cloud require a very much alike foundation.

Providing this is the goal of Windows Azure. Part of the larger Windows Azure platform, Windows Azure is the basis on which a running storing data and applications in the cloud stands. Figure 3 illustrates this idea [9]. 


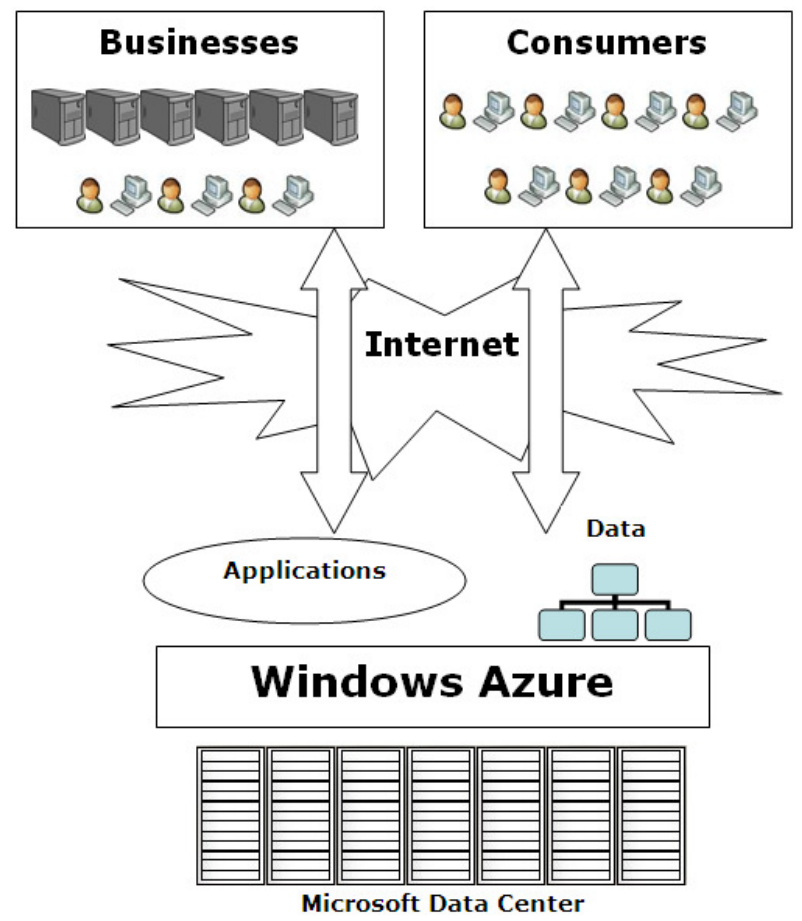

Figure 3. Windows Azure applications run in Microsoft data centers and are accessed via the Internet.

Rather than making software available that Microsoft customers can install and run themselves on their own computers, Windows Azure today is a service: Customers utilise it to operate applications and store data on Internet-accessible machines belonging to Microsoft. Those applications might make services available to businesses, to consumers, or both. Here are some examples of different kinds of applications that can be incorporating as part of Windows Azure [9]:

1. An independent software vendor (ISV) could generate an application to reach business users, an approach that's in many cases mentioned as Software as a Service (SaaS). Windows Azure was planned to support Microsoft's own SaaS applications, so ISVs can also use it as a basis for a different kind of business-oriented cloud software.

2. An ISV might produce a SaaS application that makes a target of consumers rather than businesses. Because Windows Azure is planned to maintain very scalable software, a business that decides to focus on a big market such as consumer market, might well select it as a platform for a new application.

3. Enterprises will be able to use Windows Azure to create and run applications which is going to be used by their own employees. While this case possibly won't need the huge scale of a consumer-facing application, the ability of Windows Azure to perform its required functions under stated conditions for a specified period of time and capability of being manage or controlled, could still make it an attractive choice.

To support cloud applications and data, Windows Azure has five components, as Figure 4 shows [9]. 


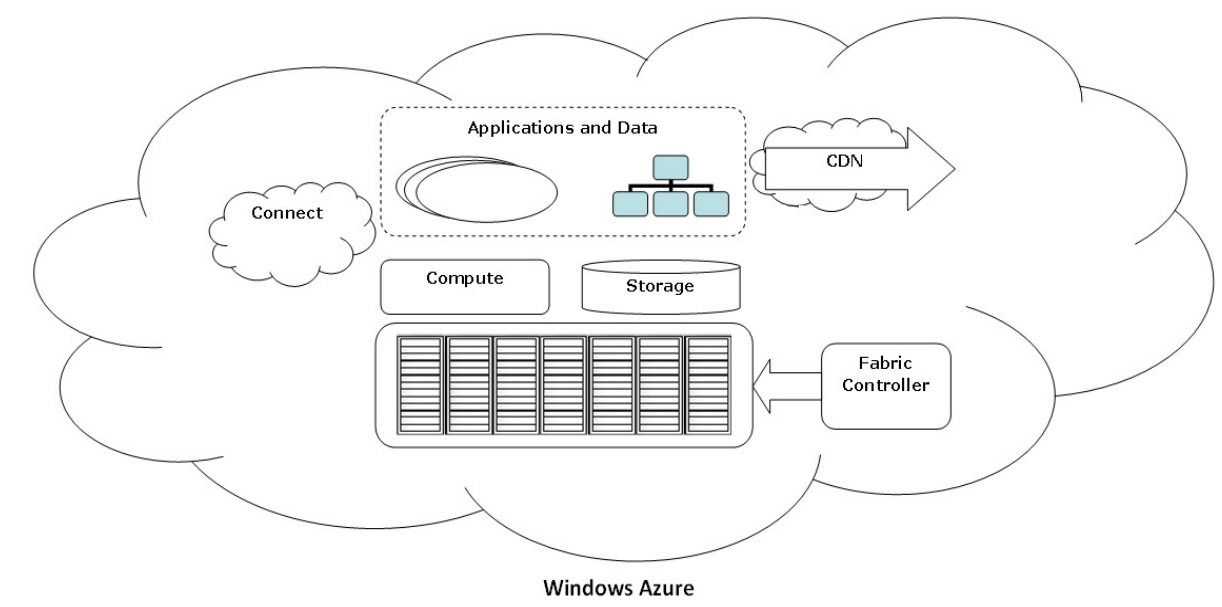

Figure 4. Windows Azure has five main parts: Compute, Storage, the Fabric Controller, the $\mathrm{CDN}$, and Connect

Those components are:

1. Compute: runs applications in the cloud. Those applications largely see a Windows Server environment, although the Windows Azure programming model isn't exactly the same as the on-premises Windows Server model.

2. Storage: stores binary and structured data in the cloud.

3. Fabric Controller: deploys, manages, and monitors applications. The fabric controller also handles updates to system software throughout the platform.

4. Content Delivery Network (CDN): speeds up global access to binary data in Windows Azure storage by maintaining cached copies of that data around the world.

5. Connect: allows creating IP-level connections between on-premises computers and Windows Azure applications.

\section{The Future of Cloud Computing}

There was a study made by the Pew Research Center's Internet \& American Life Project and Elon University's Imagining the Internet Center. That study shows that around $71 \%$ agreed with the statement: "By 2020, most people won't do their work with software running on a generalpurpose PC. Instead, they will work in Internet-based applications such as Google Docs, and in applications run from smart phones. Aspiring application developers will develop for Smartphone vendors and companies that provide Internet-based applications, because most innovative work will be done in that domain, instead of designing applications that run on a PC operating system."

On the opposite side, around 27\% agreed with the statement: "By 2020, most people will still do their work with software running on a general-purpose PC. Internet-based applications like Google Docs and applications run from smart phones will have some functionality, but the most innovative and important applications will run on (and spring from) a PC operating system. Aspiring application designers will write mostly for PCs." 
Most of those opinions, investigated by asking group of people questions, noted that cloud computing is going on to enlarge and coming to control information proceedings because it presents a lot of advantages, giving users the ability to access tools and information which they need anywhere and anytime very easy, instant, and individualized from any networked device. In addition, many experts believed that people in technology rich environments will be able to access to very complex and complicated but affordable local networks that give them permissions to have the cloud in their homes.

Most of the experts believed that people like to have many and more options of using different devices to be able to access data and applications, and - in addition to the many refers that smart phones motivating the move to the cloud and some referred to a future featuring so many types of networked appliances. A few referred the internet involved in everything in which almost every objects have a unique IP addresses and can help to be tied together as people now a day's tied together by the internet [10].

\section{Conclusion}

Cloud Computing is giving favorable promise that serves as a pattern or model help IT services to be delivered as computing utilities. The way how clouds designed helps in making services available to external users; providers need to be recompense for sharing their capabilities and resources.

Virtualization is the one of most important thing in clouds technology, because many of features such as, sharing resources on demand, security by isolation, etc based on virtualization. In addition, usability is considered as an important property of clouds. Moreover, enhancing of security is much recommended so that any enterprises could rely sensitive data on the cloud infrastructure.

Regarding clouds definitions, there is no clear and complete definition in the literature yet, which is an important task that is going to help in finding new application domains and determining the areas for the usage of the clouds.

\section{REFERENCES}

[1] Marc Fouquet, Heiko Niedermayer, and Georg Carle. Cloud Computing for the Masses. In Proceedings of the 1st ACM Workshop on User-provided Networking, co-located with CoNEXT 2009, Rome, Italy, December 2009.

[2] Amazon Web Services. http://aws.amazon.com/.

[3] Google App Engine. http://code.google.com/intl/en/appengine/.

[4] L. M. V. Gonzalez, L. R. Merino, J. Caceres, and M. Lindner. "A break in the clouds: towards a cloud definition". Computer Communication Review, 39(1):50-55, 2009

[5] Ian Foster. What is the grid? - a three point checklist.GRIDtoday, (6), July 2002.

[6] Members of EGEE-II. An egee comparative study: Grids and clouds - evolution or revolution. Technical report, Enabling Grids for E-sciencE Project, June 2008.

[7] Shantenu Jha, Andre Merzky, and Geoofrey Fox. Using clouds to provide grids higher-levels of abstraction and explicit support for usage modes. Technical report, Open Grid Forum, April 2008. 
International Journal of Distributed and Parallel Systems (IJDPS) Vol.3, No.4, July 2012

[8] Bhaskar Prasad Rimal, Eunmi Choi, Ian Lumb, "A Taxonomy and Survey of Cloud Computing Systems," ncm, pp.44-51, 2009 Fifth International Joint Conference on INC, IMS and IDC, 2009

[9] Microsoft Windows Azure. http://www.microsoft.com/enus/cloud/developer/resource.aspx?lang=java\&resourceId=introducing-windowsazure \&fbid=f5B w91FNuZi\&WT.srch=1\&WT.mc_id=1F721238-A942-40E8-BDE3545F8D90DB1D\&CR_SCC=200028940

[10] The Future of Cloud Computing. http://pewresearch.org/pubs/1623/future-cloud-computingtechnology-experts

[11] Shufen Zhang; Shuai Zhang; Xuebin Chen; Shangzhuo Wu; , "Analysis and Research of Cloud Computing System Instance," Future Networks, 2010. ICFN '10. Second International Conference on , vol., no., pp.88-92, 22-24 Jan. 2010

[12] Tharam Dillon, Chen Wu, Elizabeth Chang, "Cloud Computing: Issues and Challenges," aina, pp.27-33, 2010 24th IEEE International Conference on Advanced Information Networking and Applications, 2010

[13] Naidila Sadashiv, S.M Dilip Kumar, "Cluster, Grid and Cloud Computing: A Detailed Comparison" ICCSE 2011, pp.477-482, The 6th International Conference on Computer Science \& Education August 3-5, 2011.

[14] Shuai Zhang, Shufen Zhang, Xuebin Chen, Xiuzhen Huo. "The Comparison between Cloud Computing and Grid Computing", 2010 International Conference on Computer Application and System Modeling (ICCASM 2010), 978-1-4244-7237-6/ 2010 CIEEE.

[15] Luis M. Vaquero, Luis Rodero-Merino, Rajkumar Buyya: Dynamically scaling applications in the cloud. Computer Communication Review 41(1): 45-52 (2011)

[16] Guilherme Sperb Machado, David Hausheer, Burkhard Stiller, "Considerations on the Interoperability of and between Cloud Computing Standards," Proc. of 27th Open Grid Forum (OGF27), G2C-Net Workshop: From Grid to Cloud Networks, Oct. 2007

\section{Authors}

Dr. Elleithy is the Associate Dean for Graduate Studies in the School of Engineering at the University of Bridgeport. He has research interests are in the areas of network security, mobile communications, and formal approaches for design and verification. He has published more than one hundred fifty research papers in international journals and conferences in his areas of expertise.

Dr. Elleithy is the co-chair of the International Joint Conferences on Computer, Information, and Systems Sciences, and Engineering (CISSE). CISSE is the first Engineering/Computing and Systems Research E-Conference in the world to be

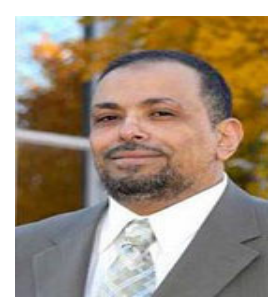
completely conducted online in real-time via the internet and was successfully running for four years. Dr. Elleithy is the editor or co-editor of 10 books published by Springer for advances on Innovations and Advanced Techniques in Systems, Computing Sciences and Software.

Munther Abualkibash is a $\mathrm{PhD}$ student in Computer Science and Engineering at the University of Bridgeport. He received his master degree from the University of Bridgeport in 2008. His current research interests image processing, programming and parallel programming.

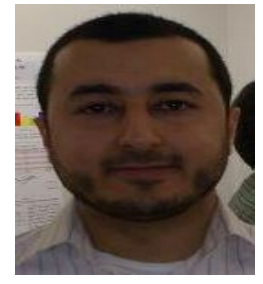

